\title{
Prevalence and clinical features of abdominal migraine compared with those of migraine headache
}

\author{
Ishaq Abu-Arafeh, George Russell
}

\begin{abstract}
The prevalence and clinical features of migraine headache and abdominal migraine were studied in the well defined population of Aberdeen schoolchildren. Ten per cent of all children (2165) aged 5-15 years were given a questionnaire inquiring, among other symptoms, about the history of headache and abdominal pain over the past year. A total of 1754 children $(81 \%)$ responded. Children with at least two episodes of severe headache and/or severe abdominal pain, attributed by the parents either to unknown causes or to migraine, were invited to attend for clinical interview and examination.

After interview, 159 children fulfilled the International Headache Society's criteria for the diagnosis of migraine and 58 children had abdominal migraine giving estimated prevalence rates of $10.6 \%$ and $4 \cdot 1 \%$ respectively. Children with abdominal migraine had demographic and social characteristics similar to those of children with migraine. They also had similar patterns of associated recurrent painful conditions, trigger and relieving factors, and associated symptoms during attacks. The similarities between the two conditions are so close as to suggest that they have a common pathogenesis. (Arch Dis Child 1995; 72: 413-417)
\end{abstract}

Keywords: abdominal migraine, migraine, recurrent abdominal pain.

Recurrent abdominal pain is common in children, and the reported prevalence rates in population based studies have varied between 6 and $15 \% .{ }^{1-3}$ Long term (5-10 years) follow up studies of such children suggest a benign prognosis in the majority. ${ }^{45}$ Recurrent abdominal pain disappears in about half the children, persists in a quarter, and is replaced by other painful symptoms (mainly headache) in the remaining quarter.

Potentially identifiable causes of recurrent abdominal pain include constipation, food or lactose intolerance, gastro-oesophageal reflux, peptic ulceration, duodenitis, Crohn's disease, urinary tract infections, and menstrual disorders. ${ }^{67}$ In these children, the pain is usually located away from the midline and may radiate or shift to other parts of the abdomen and may be burning in nature, and may be associated with or followed by other relevant clinical features of the underlying disorder. Detailed clinical history, full physical examination, and appropriate investigation are therefore important in the assessment of the child with recurrent abdominal pain, and must be included in any study of the epidemiology of this symptom.

In a large subgroup of children with recurrent abdominal pain, the abdominal pain is characteristically severe enough to disturb the child's normal daily activities, is dull or colicky in nature, and is either centred around the umbilicus or poorly localised without shift or radiation, each attack lasting for up to 72 hours. It may be associated with anorexia, nausea, vomiting, headache, and pallor. No cause for the abdominal pain can be readily identified and the pain resolves completely between attacks. Despite the recurrent nature of the condition, the children are healthy with normal growth and development. ${ }^{189}$ Because no obvious organic cause can be found, the condition has been termed psychogenic abdominal pain, ${ }^{10}$ although no psychological differences have been found between these children and pain free control children ${ }^{11}$ or children with known organic abdominal pain with a known organic cause. ${ }^{12}$ In 1986 , Symon and Russell described a group of children with otherwise unexplained severe recurrent abdominal pain who fulfilled this description and whom they considered to be cases of abdominal migraine or migraine equivalent. ${ }^{13}$ More recently they showed that the disease is favourably influenced by antimigraine treatment. ${ }^{14}$

The concept of abdominal migraine as a disease entity has been debated since the start of the century. In 1922, Brams described in detail three adult patients with abdominal migraine defined as a disorder comprising personal or family history of typical migraine headache, attacks of epigastralgia with nausea and vomiting of bile and mucus, and occasionally with diarrhoea in the absence of any definite abnormal signs on physical examination. ${ }^{15}$ The attacks lasted for a few days and began and ended rather abruptly with complete resolution between attacks. In 1933 Wyllie and Schlesinger reported on their experience with children suffering from recurrent abdominal pain as part of the periodic syndrome, and made a positive association between the syndrome and migraine. ${ }^{16}$ In 1963 Cullen and McDonald in Western Australia studied the prevalence of recurrent abdominal pain in children, in the context of the periodic syndrome, and they also noted the association of the periodic syndrome with migraine, but
Department of Child Health, University of Aberdeen, AB9 2ZD.

Accepted 14 February 1995 
Table 1 Questions relating to headache and abdominal pain

Headache

- Has your child had headache over the past year?

- If yes, were any of these severe enough to stop normal activities?

- If yes, how many times has he/she had severe headache in the past year?

- Was there a cause for each of these severe headaches?

- If yes, what was the cause for each?

Abdominal pain

- Has your child had tummyache over the past year?

If yes, were any of these severe enough to stop normal activities?

- If yes, how many times has he/she had severe tummyache in the past year?

in the past year?

If yes, what was the cause for each?

made no attempt to study that association any further. ${ }^{17}$

Among the few available population based studies of recurrent abdominal pain in children, Mortimer et al studied the epidemiology and clinical features of abdominal migraine as a clinical entity in a general practice population and found a prevalence rate of $2 \cdot 4 \%$ in children 3-11 years of age. ${ }^{18}$

We studied the epidemiology of recurrent painful conditions in Aberdeen schoolchildren aged 5 to 15 years with special emphasis on headache, migraine (as defined by the International Headache Society, IHS), recurrent abdominal pain, and abdominal migraine (defined by criteria adapted from those of Symon and Russell ${ }^{13}$ ).

\section{Population and methods}

We studied the prevalence and causes of headache and abdominal pain in the defined population of Aberdeen schoolchildren. Our results for headache and migraine have been reported previously. ${ }^{19}$ We used similar methods, in the same population, to study the prevalence and causes of abdominal pain with special emphasis on abdominal migraine. This study was approved by the local ethical committee, the Department of Education, and the community child health service (school health service).

\section{Table 2 IHS criteria for the diagnosis of migraine}

Migraine without aura (diagnostic criteria)

(A) At least five attacks fulfilling B-D

(B) Headache lasting 4-72 hours (2-48 in children)

(C) Headache has at least two of the following characteristics:

1. Unilateral location

3. Moderate or severe intensity (inhibits or prohibits daily activities)

4. Aggravation by walking stairs or similar routine physical activity

(D) During headache at least one of the following

1. Nausea and/or vomiting

1. Nausea and/or vomiting
2. Photophobia and phonophobia

Migraine with aura (diagnostic criteria):

Migraine with aura (diagnostic criteria):
(A) At least two attacks fulfilling B

At least three of the following four characteristics:

1. One or more fully reversible aura symptoms indicating focál cerebral cortical and/or brain stem dysfunction

2. At least one aura symptom develops gradually over more than four minutes or two or more symptoms occur in succession

3. No aura symptom lasts more than 60 minutes. If more than one aura symptom is present, accepted duration is proportionally increased

4. Headache follows aura with a free interval of less than 60 minutes. (It may also begin before or simultaneously with the aura)
POPULATION

As described previously, $10 \%$ of the 21607 children (5-15 years old) attending 67 schools in the city of Aberdeen were selected at random from the schools' lists, without our prior knowledge of their health or social background. ${ }^{19}$ One secondary school and its feeder primary schools were studied at one given time.

\section{QUESTIONNAIRE}

The selected children were given a questionnaire with a covering letter about the aims and nature of the study. They were asked to complete the questionnaire at home with the assistance of their parents. The questionnaire recorded the children's date of birth, sex, address, and the date of completing the questionnaire. The questions related to headache and abdominal pain are given in table 1. Children who did not return the complete questionnaire within two weeks were sent a reminder by post to their home addresses.

\section{INTERVIEWS}

Symptomatic children and their parents were invited to attend for clinical interview and examination at the school medical room if their answers showed that: (a) they had had at least two episodes of headache and/or abdominal pain severe enough to interfere with normal activities over the past year and (b) the episodes of pain were not attributed to an infectious illness, trauma, or a specific illness. For each symptomatic child identified, an asymptomatic control child matched for age and sex was also invited. Detailed present and past clinical history and family history were obtained, and a full systematic physical examination was performed.

\section{DIAGNOSIS}

The diagnosis of childhood migraine was based on the criteria of the IHS ${ }^{20}$ (table 2) and the diagnosis of abdominal migraine was based on criteria adapted from Symon and Russell ${ }^{13}$ (table 3).

\section{STATISTICAL ANALYSIS AND ESTIMATION OF} PREVALENCE RATES

To ensure standardised computer entry, data were collected on a coded checklist. SPSS for Windows $^{21}$ and confidence interval analysis ${ }^{22}$ were used. The estimated prevalence rates were calculated by adjusting for the children

Table 3 Criteria for the diagnosis of abdominal migraine

1. Pain is severe enough to interfere with normal daily activities

2. Pain is dull or colicky in nature

3. Periumbilical or poorly localised

4. Associated with any two of the following:
(A) Anorexia
(B) Nausea
(C) Vomiting
(D) Pallor

5. Each attack lasts for at least one hour

6. Complete resolution of symptoms between attacks 
Table 4 Causes of severe headache and severe abdominal pain as attributed by parents; results are number (\%)

\begin{tabular}{lll}
\hline Cause & Severe headache & Severe abdominal pain \\
\hline Not known & $164(42)$ & $173(31)$ \\
Infections & $121(31)$ & $270(48)$ \\
Migraine & $59(15)$ & $11(2)$ \\
Trauma & $20(5)$ & 0 \\
Specific cause & $9(2)$ & $75(13) \dagger$ \\
Constipation & 1 & $21(4)$ \\
Others & $17(4)$ & $16(3)$ \\
Total & 391 & 566 \\
\hline
\end{tabular}

«Upper respiratory tract infections, viral infections, and gastroenteritis.

†Menstrual periods in $\mathbf{5 4}$ and related to food in 21 .

who had severe recurrent headache or abdominal pain, but failed to attend for clinical interview. Therefore, the actual prevalence rate for migraine was multiplied by a factor of $1 \cdot 17$ (241 children invited to attend for interview divided by 206 who actually attended), and similarly by a factor of $1 \cdot 24$ for children with abdominal migraine ( 138 children invited divided by 111 attended).

\section{Results}

QUESTIONNAIRE

Completed questionnaires were returned by 1754 children (81\%), of whom $153(7 \%)$ responded after a reminder. There were 888 boys $(50 \cdot 6 \%)$ and 866 girls $(49 \cdot 4 \%)$. Their mean (SD) age was $10.2(3.0)$ years. The parents of 30 children declined to participate.

A total of 1263 children $(72 \%)$ had at least one episode of abdominal pain over the past year and 566 of them (32\%) had pain severe enough to interfere with normal activities, compared with 1166 children $(66 \%)$ who had at least one episode of headache and 391 $(22 \%)$ who had severe headache. The causes to which parents attributed severe headache and abdominal pain are shown in table 4 . Of these, 138 children had at least two episodes of severe abdominal pain (prevalence rate $7.9 \%$, $95 \%$ confidence intervals (CI) $6 \cdot 7$ to $9 \cdot 2$ ) and 241 children had at least two episodes of severe headache $(13 \cdot 7 \%, 95 \%$ CI $12 \cdot 1$ to $15 \cdot 4)$ for which no specific causes were given by parents or were attributed to migraine.

\section{INTERVIEWS}

Three hundred and seventy nine children fulfilling the criteria detailed under methods (241 with severe headache and 138 with

Table 5 Estimated age and sex prevalence rates of abdominal migraine

\begin{tabular}{|c|c|c|c|c|c|}
\hline \multirow{2}{*}{$\begin{array}{l}\text { Age } \\
\text { (years) }\end{array}$} & \multicolumn{2}{|l|}{ Boys } & \multicolumn{2}{|l|}{ Girls } & \multirow{2}{*}{$\begin{array}{l}\text { Total } \\
\text { estimated } \\
\text { prevalence (\%) }\end{array}$} \\
\hline & Population & No (\%) & Population & No (\%) & \\
\hline $\begin{array}{r}5 \\
6 \\
7 \\
8 \\
9 \\
10 \\
11 \\
12 \\
13 \\
14 \\
15\end{array}$ & $\begin{array}{l}96 \\
86 \\
84 \\
96 \\
88 \\
72 \\
92 \\
87 \\
79 \\
58 \\
50\end{array}$ & $\begin{array}{l}0 \\
3(4 \cdot 3) \\
2(3 \cdot 0) \\
2(2 \cdot 6) \\
2(2 \cdot 8) \\
2(3 \cdot 4) \\
2(2 \cdot 7) \\
5(7 \cdot 1) \\
4(6 \cdot 3) \\
0 \\
0\end{array}$ & $\begin{array}{r}78 \\
87 \\
85 \\
80 \\
80 \\
81 \\
103 \\
78 \\
69 \\
65 \\
60\end{array}$ & $\begin{array}{l}3(4 \cdot 8) \\
3(4 \cdot 3) \\
4(5 \cdot 8) \\
2(3 \cdot 1) \\
4(6 \cdot 2) \\
6(9 \cdot 2) \\
2(2 \cdot 4) \\
7(11 \cdot 1) \\
3(5 \cdot 4) \\
1(1 \cdot 9) \\
1(2 \cdot 1)\end{array}$ & $\begin{array}{l}2 \cdot 1 \\
4 \cdot 3 \\
4 \cdot 4 \\
2 \cdot 8 \\
4 \cdot 4 \\
6 \cdot 5 \\
2 \cdot 5 \\
9 \cdot 0 \\
5 \cdot 9 \\
1 \cdot 0 \\
1 \cdot 1\end{array}$ \\
\hline $5-15$ & 888 & 22 & 866 & 36 & $4 \cdot 1$ \\
\hline
\end{tabular}

severe abdominal pain as their main complaint) were invited to attend for interview; 317 children (206 with headache; $85 \%$ and 111 with abdominal pain; 80\%) attended. The parents of $286(90 \%)$ were either present during the interviews or gave interviews on the telephone.

One hundred and forty five children fulfilled the IHS criteria for the diagnosis of migraine, 44 children fulfilled the criteria for the diagnosis of abdominal migraine, and 14 children fulfilled the diagnostic criteria for both conditions. Forty seven children had recurrent abdominal pain that did not fulfil our criteria for the diagnosis of abdominal migraine, although a specific diagnosis could not be made or excluded on clinical grounds alone, and six children had recurrent abdominal pain for which specific causes were identified.

\section{CHILDREN WITH MIGRAINE}

A total of 159 children, 74 boys (mean (SD) age $11 \cdot 2(2 \cdot 7)$ years) and 85 girls $(11 \cdot 8(2 \cdot 7)$ years), fulfilled the IHS criteria for the diagnosis of migraine. The epidemiological features of this group of children have been reported previously. ${ }^{19}$ Their clinical features are given below alongside those of children with abdominal migraine.

\section{CHILDREN WITH ABDOMINAL MIGRAINE}

There were 58 children with abdominal migraine: 22 boys $(10.5(2.7)$ years $)$ and 36 girls $(10.2(2 \cdot 8)$ years). The estimated prevalence rate of abdominal migraine is $4 \cdot 1 \%(95 \%$ CI $3 \cdot 2$ to $5 \cdot 2)$. Age and sex specific prevalence rates of abdominal migraine are presented in table 5. Symptoms were reported to have started at all ages from the first year of life with a mean (SD) age of onset of $7 \cdot 0(3 \cdot 2)$ years and with two peaks at 5 and 10 years, similar to childhood migraine (table 5).

The abdominal pain was described as 'just sore' or dull in 35 children (60\%), colicky in 13 $(22 \%)$, sharp in eight (14\%), and other descriptions in two. Pain was periumbilical in 45 children $(78 \%)$ and diffused over the whole abdomen in nine $(16 \%)$, but four children were unable to give a precise siting. The abdominal pain was severe enough to inhibit all or most normal daily activities in 42 children ( $72 \%$ ) but permitted some activity in $16(28 \%)$. Before the onset of the abdominal pain, eight children (14\%) experienced non-specific prodromal symptoms such as behaviour and mood changes, headache, and anorexia.

Attacks of abdominal pain occurred on a mean (SD) of $14(22.4)$ times/year (range 2-100) with each attack lasting for a mean of $17(18 \cdot 1)$ hours (range 1-72). Twenty children (34\%) had at least one first degree relative with migraine (as defined by the IHS), compared with $75(47 \%)$ children with migraine and only 10 control children $(17 \%)$ matched for age and sex.

Children with abdominal migraine lost a mean (SD) of $3.7(4.8)$ school days/year (range $0-36$ ) due to abdominal pain and a mean of 
Table 6 Demographic features of children with migraine and abdominal migraine

\begin{tabular}{lcc}
\hline & $\begin{array}{c}\text { Migraine } \\
(n=159)\end{array}$ & $\begin{array}{l}\text { Abdominal migraine } \\
(n=58)\end{array}$ \\
\hline Male:female ratio & $1: 1 \cdot 1$ & $1: 1 \cdot 6$ \\
Mean (SD) age in years & $11 \cdot 5(2 \cdot 7)$ & $10 \cdot 3(2 \cdot 8)$ \\
Peak age of prevalence in years & $12.3(5$ and 10$)$ & $12(5$ and 10$)$ \\
Mean age of onset in years (peaks) & $85(47)$ & $20(34)$ \\
Migraine in first degree relatives $(\%)^{\star}$ & 75 & 20 \\
\hline
\end{tabular}

^At least one first degree relative had migraine fulfilling IHS criteria.

$6 \cdot 8(7 \cdot 1)$ days/year (range $0-36$ ) due to illnesses, including abdominal pain, compared with a mean of $3.1(4.0)$ days/year (range $0-20$ ) in the matching group of control children (mean difference between pairs $=3 \cdot 7,95 \%$ CI $1 \cdot 4$ to $6 \cdot 0, p=0 \cdot 002$ ).

Children with abdominal migraine, compared with children with migraine fulfilling the IHS criteria, have similar demographic features (table 6), associated recurrent conditions (table 7), precipitating trigger factors (table 8), associated symptoms during attacks (table 9), and relieving factors (table 10).

\section{Discussion}

The response rate to the screening questionnaire $(81 \%)$ and the attendance rate at the clinical interviews $(80 \%)$ were high enough to allow for a reliable assessment of the prevalence rates of headache, migraine, recurrent abdominal pain, and abdominal migraine. Our study has confirmed the high prevalence of headache (66\%) and abdominal pain $(70 \%)$ in children between 5 and 15 years of age. Also it has shown that a high proportion of children have severe symptoms; $32 \%$ and $22 \%$ of all children had abdominal pain and headache, respectively, that were severe enough to stop normal activities. In about one third of children with severe headache and in about half the children with severe abdominal pain, parents attributed the symptoms to upper respiratory tract infections, viral illnesses, or gastroenteritis. However, $7 \cdot 9 \%$ of all the children had at least two episodes of severe abdominal pain over the previous year for which the parents were unable to find a cause. This finding is consistent with the reported prevalence rates of recurrent abdominal pain in the literature. ${ }^{1-3} 17$

Abdominal migraine as a clinical diagnosis continues to be a subject of controversy, ${ }^{23-25}$ and is not yet included in the diagnostic classification of migraine by the IHS. ${ }^{20}$ In this study we based our diagnosis of abdominal migraine on diagnostic criteria as selective as possible, derived from published data and from our own

Table 7 Associated recurrent painful conditions; results are number (\%)

\begin{tabular}{lcc}
\hline & $\begin{array}{c}\text { Migraine } \\
(n=159)\end{array}$ & $\begin{array}{c}\text { Abdominal migraine } \\
(n=58)\end{array}$ \\
\hline Migraine headache only & $106(67)$ & 0 \\
Non-migraine headache & 0 & $4(7)$ \\
Abdominal migraine only & 0 & $32(55)$ \\
Non-specific abdominal pain & $12(8)$ & 0 \\
Both migraine and abdominal migraine & $14(9)$ & $14(24)$ \\
Associated paroxysmal vertigo & $14(9)$ & $3(5)$ \\
Associated limb pain & $9(6)$ & $3(5)$ \\
Associated cyclical vomiting & $3(2)$ & $1(2)$ \\
Other combinations & 1 & $1(2)$
\end{tabular}

Table 8 Precipitating factors of attacks of migraine and abdominal migraine; results are number (\%)

\begin{tabular}{llc}
\hline & $\begin{array}{c}\text { Migraine } \\
(n=159)\end{array}$ & $\begin{array}{c}\text { Abdominal migraine } \\
(n=58)\end{array}$ \\
\hline Stress & $53(33)$ & $15(26)$ \\
Tiredness & $40(25)$ & $13(22)$ \\
Travel & $32(20)$ & $9(16)$ \\
Bright light & $31(19)$ & 0 \\
Lack of sleep & $30(18)$ & $3(1 \cdot 5)$ \\
Certain foods & $23(14)$ & $5(9)$ \\
Hot climate & $23(14)$ & $1(0 \cdot 5)$ \\
Missing a meal & $19(12)$ & $10(17)$ \\
\hline
\end{tabular}

clinical experience with a large number of children attending our outpatient clinics with recurrent abdominal pain. By using these criteria we excluded (a) all children who had burning abdominal pain that might indicate gastro-oesophageal reflux or peptic ulceration, (b) all children with non-midline abdominal pain which might indicate large bowel, renal, or menstrual disorder, (c) all children with mild symptoms which did not interfere with normal daily activities, (d) all children with attacks of less than one hour's duration, and (e) all children who despite fulfilling the above criteria also had problems with food intolerance, malabsorption, weight loss, or persistent symptoms between attacks of abdominal pain. In order to avoid selection bias, a family or personal history of migraine or any other painful condition was not included in the criteria for the diagnosis of abdominal migraine. Until an objective test for the diagnosis of migraine headache and abdominal migraine is available, it will continue to be necessary to use such restrictive criteria and to keep these children under a long period of follow up, to ensure the complete absence of other alternative causes of recurrent abdominal pain. The appropriate use of laboratory tests, the regular review of the assumed diagnosis, and long term follow up are essential for the safe and accurate use of such diagnostic criteria.

The estimated prevalence rate of abdominal migraine $(4 \cdot 1 \%)$ in this well defined childhood population is the first such report in the literature. However, in a study related exclusively to a single general practice, Mortimer et al reported a comparable prevalence rate of $2 \cdot 4 \%$ in children aged between 3 and 11 years. ${ }^{18} \mathrm{We}$ are unaware of any published data on children up to the age of 15 years.

Our study shows that the clinical features of abdominal migraine are similar in many respects to those of childhood migraine. In common with childhood migraine, ${ }^{26}$ abdominal migraine is commoner in girls than in boys

Table 9 Symptoms associated with attacks of migraine and abdominal migraine; results are number (\%)

\begin{tabular}{lcc}
\hline & $\begin{array}{c}\text { Migraine } \\
(n=159)\end{array}$ & $\begin{array}{c}\text { Abdominal migraine } \\
(n=58)\end{array}$ \\
\hline Anorexia & $143(90)$ & $53(91)$ \\
Nausea & $136(86)$ & $53(91)$ \\
Vomiting & $89(56)$ & $42(72)$ \\
Pallor & $140(88)$ & $52(90)$ \\
Feeling unwell & $134(84)$ & $49(85)$ \\
Light intolerance & $123(77)$ & 1 \\
Noise intolerance & $109(69)$ & $4(7)$ \\
Vertigo & $113(71)$ & $12(21)$ \\
Feeling hot & $51(32)$ & $19(33)$ \\
\hline
\end{tabular}


Table 10 Relieving factors of attacks of migraine and abdominal migraine; results are number (\%)

\begin{tabular}{lcc}
\hline & $\begin{array}{c}\text { Migraine } \\
(n=159)\end{array}$ & $\begin{array}{l}\text { Abdominal migraine } \\
(n=58)\end{array}$ \\
\hline Rest & $156(98)$ & $51(88)$ \\
Sleep & $141(89)$ & $37(64)$ \\
Simple analgesics & $129(81)$ & $22(38)$ \\
Vomiting & $20(13)$ & $13(22)$ \\
Eating & $5(3)$ & $3(5)$ \\
Others & $19(12)$ & $8(14)$ \\
\hline
\end{tabular}

(female:male ratio $1 \cdot 16: 1$ ) and starts at a mean age of 7.0 years with two distinct peaks at the ages of 5 and 10 years. The reasons for the two peaks are uncertain, but it is possible that school entrance at the age of 5 years, representing a major stressful life event for the child, is responsible for the first peak. Also of interest is the high number of children who had concurrently both migraine headache and abdominal migraine. The prevalence rate of migraine headache in children with abdominal migraine $(24 \%)$ is just over twice the prevalence rate of migraine in the general childhood population $(10.6 \%)$, and conversely the prevalence rate of abdominal migraine in children with migraine (9\%) is just over twice the prevalence of abdominal migraine in the general childhood population $(4 \cdot 1 \%)$. Other associated recurrent conditions, and family history of migraine, are similar in both groups of children. The prevalence of migraine in first degree relatives was twice as common in children with abdominal migraine as in control healthy children. The relatively low prevalence rates of migraine in the first degree relatives (34\% in children with abdominal migraine and $17 \%$ in controls) may be due to the fact that most of the siblings of the children studied are too young and consist of a low prevalence childhood population.

Attacks of abdominal pain were very similar to attacks of migraine headache in having common trigger and relieving factors, and associated gastrointestinal, sensory, and vasomotor symptoms. Attacks of abdominal migraine in this population based study were comparable in frequency (mean 14/year) and duration of symptoms (mean 17 hours) with those found in a general practice childhood population (13.5/year and 13.3 hours respectively), ${ }^{18}$ but less frequent and of shorter duration than in children attending hospital outpatient clinics. ${ }^{13}$

The effects of abdominal migraine on social life and education of the affected children are difficult to quantify in isolation from other factors, but abdominal migraine was associated with a considerable number of days of school absence because of the recurrent abdominal pain (a mean of 3.7 days/year).
In conclusion, there is increasing evidence from studies in paediatric ${ }^{1318}$ and adult ${ }^{25}$ patients that abdominal migraine is a separate entity that can be distinguished from other causes of recurrent abdominal pain. With a prevalence of around $4 \%$ in 5-15 year old children, it is clearly a sizeable clinical problem, and a significant cause of recurrent abdominal pain. With effective prophylaxis now available, ${ }^{14}$ it is important to use strict diagnostic criteria to make a positive diagnosis.

IAA was supported by a clinical fellowship from Glaxo for her advice on statistical analysis and to Mrs Connie Durno for help with data entry.

1 Apley J, Naish N. Recurrent abdominal pains: a field survey of 1000 school children. Arch Dis Child 1958; 33: 165-70.

2 Pringle MLK, Butler NR, Davie R. 11000 seven-year-olds. London: Longman, 1966: 184.

3 Oster J. Recurrent abdominal pain, headache and limb pains in children and adolescents. Pediatrics 1972; 50: 429-36.

4 Magni G, Pierri M, Donzelli F. Recurrent abdominal pain in children: a long term follow up. Eur $\mathcal{F}$ Pediatr 1987; 146: in child.

5 Valman HB. Recurrent abdominal pain. BMF 1981; i: 1949-51.

6 Mavromichalis I, Zaramboukas T, Richman PI, Slavin G. Recurrent abdominal pain of gastro-intestinal origin. Eur $\Im$ Pediatr 1992; 151: 560-3.

7 Van Der Meer SB, Forget PP, Kuijten RH, Arends JW. Gastroesophageal reflux in children with recurrent abdominal pain. Acta Paediatr 1992; 81: 137-40.

8 Dodge J. Recurrent abdominal pain in children. BMF 1976 i: $385-7$.

9 Vulliamy DG. Recurrent abdominal pain and vomiting in children. BMF 1965; i: 1113-5.

10 Nicol AR. Psychogenic abdominal pain in children. $\mathrm{Br} F$ Hosp Med 1982; 27: 351-3.

11 McGrath PJ, Goodman JT, Firestone P, Shipman R, Peters S. Recurrent abdominal pain: a psychogenic disorder. S. Recurrent abdominal pain: a

12 Raymer D, Weininger O, Hamilton JR. Psychological problems in children with abdominal pain. Lancet 1984; i 439-40.

13 Symon DNK, Russell G. Abdominal migraine: a syndrome defined. Cephalalgia 1986; 6: 223-8.

14 Symon DNK, Russell G. Double blind placebo controlled trial of pizotifen syrup in the treatment of abdomina migraine. Arch Dis Child 1995; 72: 48-50.

15 Brams WA. Abdominal migraine. $\mathcal{F} A M A$ 1922; 78: 26-7. 6 Wyllie WG, Schlesinger B. The periodic group of disorders in childhood. British fournal of Children's Diseases 1933; XXX: $349-51$.

17 Cullen KJ, MacDonald WB. The periodic syndrome: its nature and prevalence. Med $\mathcal{F}$ Aust 1963; ii: 167-72.

18 Mortimer MK, Kay J, Jaron A. Clinical epidemiology of childhood abdominal migraine in an urban general childhood abdominal migraine in an urban

19 Abu-Arafeh IA, Russell G. Prevalence of headache and migraine in schoolchildren. BMF 1994; 309: 765-9.

20 Headache Classification Committee of the International Headache Society. Classification and diagnostic criteria for headache disorders, neuralgias and facial pain. Cephalalgia 1988; 8 (suppl 7): 1-96.

21 SPSS. SPSS for Windows. Release 5.0.2. Chicago: SPSS Inc, 1993.

22 Gardner SB, Winter PD, Gardner M. Confidence interval analysis version 1.0. London: BMJ, 1989.

23 Symon DNK. Is there a place for 'abdominal' migraine as a separate entity in the IHS classification? Yes! Cephalalgia 1992; 12: 345-6.

24 Hockaday JM. Is there a place for 'abdominal' migraine as a separate entity in the IHS classification? No! Cephalalgia 1992; 12: 346-8.

25 Axon ATR, Long DE, Jones SC. Abdominal migraine: does it exist? [Editorial.] $\mathcal{F}$ Clin Gastroenterol 1991; 13: 615-6. 26 Abu-Arafeh IA, Russell G. Prevalence and clinical spectrum of childhood migraine. Cephalalgia 1993; 13 (suppl 13):
209. 